\title{
Anticoagulant effects of different calcium supplementation pathways in calcium-free RCA- CWVH: a study protocol for a prospective randomized cross-over trial
}

Biantong JIANG ( $\nabla 219717102 @ 163 . c o m)$

The First Hospital of Lanzhou University https://orcid.org/0000-0002-1136-469X

\section{Zhigang ZHANG}

The First Hospital of Lanzhou University

\section{Xiu JIN}

The First Hospital of Lanzhou University

Haiye WANG

The First Hospital of Lanzhou University

\section{Yuchen WU}

The First Hospital of Lanzhou University

\section{Caiyun ZHANG}

The First Hospital of Lanzhou University

\section{Method Article}

Keywords: Continuous venous-venous hemofiltration, regional citrate anticoagulation, calcium, effect, safety, cross-over trial

Posted Date: January 31st, 2019

DOI: https://doi.org/10.21203/rs.2.264/v1

License: (c) (1) This work is licensed under a Creative Commons Attribution 4.0 International License. Read Full License 


\section{Abstract}

Background When regional citrate anticoagulation used in continuous renal replacement therapy, one of the key aspects to achieve safe and effective extracorporeal circulation is the management of calcium ions. For calcium-free RCA-CVVH, the anticoagulant effects of different calcium supplementation pathways have not yet been explored. In this trial, we would test our hypothesis that compared with the SCV, when calcium was infused through the VL-FV, the arterial iCa2+ was lower.

Methods This is a prospective randomized cross-over trial involving 24 patients undergoing RCA-CVVH. The patients were randomly divided into two groups: VL-FV-SCV group and SCV-VL-FV group. The difference of iCa2+ between arterial iCa2+ and post-filtration iCa2+ was compared. Secondary indicators included the incidence rates of catheter dysfunction and hypocalcemia.

Discussion This is the first trial on the anticoagulant effects of calcium-free RCA-CVVH through different calcium supplement routes. We will confirm that the arterial iCa $2+$ level is slightly lower when calcium is infused in the VL-FV than in the SCV, and the incidence rates of catheter dysfunction and hypocalcemia will help us to determine which site is safer.

Trial Registration CHiCTR registry: ChiCTR1800020046. Registered on 12 December 2018. (http://www.chictr.org.cn/listbycreater.aspx).

Keywords: Continuous venous-venous hemofiltration, regional citrate anticoagulation, calcium, effect, safety, cross-over trial

\section{Background}

Anticoagulation in vitro is an important part of continuous renal replacement therapy (CRRT). The commonly anticoagulants in clinic are heparin, low molecular weight heparin and citrate, etc. In recent years, a good deal of studies has confirmed that citrate has the advantages of good tolerance, low risk of bleeding and good biocompatibility, as well as no effect on hemofiltration. In 2012, KDIGO recommended citrate as the first choice of anticoagulant agent in renal replacement therapy for acute kidney injury (AKI) ${ }^{[1]}$, and since then, regional citrate anticoagulation (RCA) has been widely used in clinic.

When RCA used in CRRT (Referred to as RCA-CRRT), one of the key aspects to achieve safe and effective extracorporeal circulation is the management of calcium ions. The combination of citrate and ionized calcium inhibits the coagulation process, which can achieve regional anticoagulation during the extracorporeal circulation of RCA. When the blood returns to the body, part of the citrate is removed by the filter in the form of calcium citrate chelate, and the remaining part of the systemic circulation is metabolized by the tricarboxylic acid cycle, at the same time, the calcium ions is released ${ }^{[2]}$. In a sense, if we want to ensure normal coagulation function in the body, you must supplement some of the calcium removed by the filter. 
In the process of CRRT, as long as enough calcium is added before blood transfused back into the body, the coagulation function of patients will not be affected. Meanwhile, the aim of local anticoagulation in vitro can also be achieved.

Due to the different types of dialysate and replacement fluid, the pathway of calcium supplementation is also different in clinic. A number of related foreign studies ${ }^{[3-4]}$ have shown that the appropriate ratio of calcium-containing dialysate and/or replacement fluid can be sufficient to achieve effective and safe anticoagulation and calcium supplementation, and to meet the requirements of calcium balance without additional calcium supplementation. For calcium-free RCA, additional calcium supplements are required. There are generally two ways to do this: the deep vein and the venous line ${ }^{[5-6]}$. A common clinical problem is how to choose between the two ways when calcium supplementation is given. According to the process of blood circulation, extracorporeal anticoagulation mechanism of RCA and the characteristics of blood filter catheters, etc., the two pathways are reasonable and feasible in clinic. However, whether it has effects on pre-filtration calcium ion concentration $\left(\mathrm{iCa}^{2+}\right)$, post-filtration $\mathrm{iCa}^{2+}$ or the arterial $\mathrm{iCa}^{2+}{ }^{+}$, whether it has effects on the rate of calcium supplementation and the regulation of citrate, which is still blind spots without support for relevant data in actual clinical work.

The purpose of this study is: (1) To explore the difference in anticoagulant effects of two different calcium supplementation pathways in calcium-free RCA-CRRT; (2)To explore the safety of the two pathways to increase the evidence for individualized calcium supplementation, so that clinical staff can flexibly adjust the calcium supplementation rate in actual work according to different calcium supplementation pathways.

\section{Methods}

\section{Study design}

This is a prospective, single-center, randomized cross-over trial. This study was approved by Medical Ethics Committee of the First Hospital of Lanzhou University (LDYYLL2018-165) and registered at chictr.org.cn (ChiCTR1800020046). The trial is still being conducted at the First Hospital of Lanzhou University. The study protocol is reported according to the Standard Protocol Items: Recommendations for Interventional Trials guidelines (Figure 1). In addition, the final report will adhere to the Consolidated Standards of Reported Trials (CONSORT) statement.

\section{Study population}

Adults patients (age $\geq 18$ years), who are received RCA-CVVH (Continuous venous-venous hemofiltration) with hemofiltration catheter punctured in femoral vein (FV). The exclusion criteria are patient refusal; patients with intravenous calcium or oral calcium before enrollment; patients with hyperkalemia $\left(\mathrm{iCa}^{2+}>1.35 \mathrm{mmol} / \mathrm{L}\right)$ before enrollment.

\section{Ethics, consent, and permission}


This study follows the tenets of the Declaration of Helsinki, which was approved by Medical Ethics Committee of the First Hospital of Lanzhou University, and written informed consent will be obtained from all participants.

\section{Randomization}

The trial follows the CONSORT 2010 flow chart form randomization (Fig.2). A randomization code was generated with a computerized system by the nurse who is not involved with this trial. When eligible patients enter this trial, the nurse will notify the group allocation to investigators. Based on the different calcium supplementation pathways, patients will be randomly assigned to two groups (Group A: VL-FVSCV (Subclavian vein) group; Group B: SCV-VL-FV group) with 1:1 ratio. The randomization schemes in sealed opaque envelopes.

\section{Sample size}

Based on the formula $n=\left(\mu_{a}+\mu_{\beta}\right)^{2} \sigma_{d}{ }^{2} / \delta_{d}{ }^{2}, \mu_{a}=1.960, \mu_{\beta}=1.280$. The Pre-experiment results showed that $\sigma_{d}$ $=0.038, \delta_{d}=0.028$. Considering a $10 \%$ dropout rate, 24 patients are needed for this trial.

\section{Study protocol}

\section{CRRT parameters}

CRRT are carried out using Prismaflex machines with Prismaflex M100set. 15F double-lumen hemofiltration catheters will be used for FV catheterization. Blood flow will be set at $150 \mathrm{ml} / \mathrm{min}$ $180 \mathrm{ml} / \mathrm{min}$, and a calcium-free dialysate at $2000 \mathrm{ml} / \mathrm{h}$. RCA are performed with a continuous infusion of $4 \%$ citrate into the arterial line at $160 \mathrm{ml} / \mathrm{h}-200 \mathrm{ml} / \mathrm{h}$ which is adjusted to maintain post-filtration $\mathrm{iCa}^{2+}$ at $0.25 \mathrm{mmol} / \mathrm{L} \otimes 0.45 \mathrm{mmol} / \mathrm{L}$ and calcium chloride $\left(\mathrm{CaCl}_{2}, 10 \mathrm{ml} / 0.5 \mathrm{~g}\right)$ into the venous line (Group A) or the SCV (Group B). The initial calcium infusion rate is set following the doctors' advice and adjusted to achieve the arterial $\mathrm{iCa}^{2+}$ at $1.0 \mathrm{mmol} / \mathrm{L} \otimes 1.2 \mathrm{mmol} / \mathrm{L}$ after each $\mathrm{iCa}^{2+}$ measurement by $1-2 \mathrm{ml} / \mathrm{h}$ (in accordance with clinical experience and without a pre-specified scheme). Relevant parameters (including blood flow, transmembrane pressure (TMP), ultrafiltration rate (UFR), replacement fluid rate, dosage of citrate, etc.) and initial arterial $\mathrm{iCa}^{2+}$ will be recorded at the beginning of CRRT.

\section{Calcium supplementation procedure}

When $\mathrm{CVVH}$ is started, arterial $\mathrm{iCa}^{2+}$ and post-filtration $\mathrm{iCa}^{2+}$ will be measured every $1-2 \mathrm{~h}$. The calcium infusion rate will be adjusted to achieve the desired $\mathrm{iCa}^{2+}$ target range. $\mathrm{Ca}^{2+}$ is monitored until its level became stable and then researchers continuously check the arterial $\mathrm{iCa}^{2+}$ three times under the rate remaining unchanged. At that moment, researchers will record the rate of calcium infusion and the specific time. After that, the calcium supplementation pathway should be changed from the design (Group A is to the SCV; Group B is to the venous line) holding the previous rate. After 1 hour, arterial iCa ${ }^{2+}$ 
and post-filtration $\mathrm{iCa}^{2+}$ are measured again. If phosphate and carbonate drugs are required in intravenous infusion during RCA-CVVH, they are stipulated to be transfused through peripheral veins.

\section{Withdrawal, dropout, and discontinuation}

If participants voluntarily withdraw their informed consent at any time during the study, they will be withdrawn from the trial and exclude from data analysis. The trial will be discontinued if the following events occur during the procedure: the patient dies; the patient gets better and CRRT will be stopped; the patient gives up the treatment; the patient goes out for examination; the treatment is be compelled to be quitted due to the equipment performance.

\section{Data collection}

Demographic data will be collected before CVVH. The data collected includes age, sex, diagnosis, indication for RRT, APACHE II score, mechanical ventilation. CVVH relevant parameters will also be collected, which contains blood flow, TMP, PA, PV, UFR, dosage of citrate. As the main measurement index, arterial $\mathrm{iCa}^{2+}$ and post-filtration $\mathrm{iCa}^{2+}$ will be measured by blood gas analyzer, of which the blood sample size is $1 \mathrm{~mL}$. The $\mathrm{PH}$ value, $\mathrm{K}^{+}, \mathrm{tHbc}$ and $\mathrm{iCa}^{2+}$ are also recorded the same time.

Data on related complications, including catheter dysfunction (Blood flow $<200 \mathrm{ml} / \mathrm{min}$, pre-pump arterial pressure $<-250 \mathrm{mmHg}$ or venous pressure $<250 \mathrm{mmHg}$ ) and hypocalcemia (Arterial $\mathrm{iCa}^{2+}<0.9 \mathrm{mmol} / \mathrm{L}$ ) will be collected during the procedure as secondary indicators.

\section{Data management}

The numbered data are double-input by EpiData3.1 software and managed by Excel software. During the trial, the personal information and related information of all the subjects are kept secret, which are only used for the statistical analysis of this study.

\section{Statistical analysis}

Analyses will be performed with SPSS 22.0. Calcium concentration, the main evaluating indicator, will be expressed as mean \pm standard deviation or median (interquartile range) and be compared to the twostage cross-over ANOVA or Wilcoxon signed-rank test based on results of the Shapiro-Wilk test. All categorical variables will be expressed as numbers or percent and be analyzed with the chi-square test. Multivariate linear regression will be used to analyze the influence factors of $\mathrm{iCa}^{2+}$, in which backward stepwise regression is used to screen variables, $=0.05 \rrbracket=0.10$. All statistical tests are conducted by bilateral test, and $P \leq 0.05$ is considered significant.

\section{Discussion}

This is the first prospective trial on the anticoagulant effects of different calcium supplementation pathways in calcium-free RCA-CVVH. All patients will use double-lumen hemofiltration catheters. 
In the process of CRRT, the combination of ionized calcium and citrate, infused into arterial line, inhibits the coagulation process through reducing the local free calcium, which can achieve regional anticoagulation during the extracorporeal circulation. When the blood returns to the body, part of the citrate is removed by the filter membrane with the action of convection and diffusion in the form of calcium citrate chelate, and the remaining entering the systemic circulation is metabolized into carbon dioxide and water by the tricarboxylic acid cycle in mitochondria of the liver, muscle tissue and kidney, at the same time, the calcium ions is released ${ }^{[7]}$. In a sense, if we want to ensure normal coagulation function in the body, you must supplement some of the calcium removed by the filter. In the process of CRRT, as long as enough calcium is added before blood transfused back into the body, the coagulation function of patients will not be affected. Meanwhile, the aim of local anticoagulation in vitro can also be achieved.

For patients receiving RCA-CVVH with double-lumen hemofiltration catheters, additional calcium supplements are required to ensure the safe and effective extracorporeal circulation. There are generally two ways in clinic: the deep vein and the venous line. According to the process of blood circulation, extracorporeal anticoagulation mechanism of RCA and the characteristics of blood filter catheters, etc., the two pathways are reasonable and feasible in clinic. There is a hole on each line (arterial line and venous line) of the catheter, which are located in the FV together, which could bring on a phenomenon-"recycle" [7]. If the calcium is supplied in the first way mentioned above, the adjustment of citrate may be affected by the re-entry of $\mathrm{iCa}^{2+}$ from the venous line into the arterial line to participate in the extracorporeal cyclic process. On the other hand, if the second scheme is implemented, the recycle of $\mathrm{iCa}^{2+} \mathrm{can}$ be reduced appropriately for which uses the other deep veins instead of the venous lines of dialysis catheters. We will hypothesized that compared with the deep vein, when calcium was infused through the venous line, the arterial $\mathrm{iCa}^{2+}$ was lower. We will test our hypothesis by comparing the before and after effects of calcium supplementation in two different locations (the SCV and the FV-VL).

During RCA-CVVH, poor management of calcium concentration, for instance, insufficient supplementation and citrate accumulation, may result in hypocalcemia during or after dialysis, and serious complications, such as hypocalcemia tetany, hypotension, arrhythmia, and even sudden cardiac arrest and death. On another face, excessive supplementation would lead to hyperkalemia or tissue calcium deposition ${ }^{[8]}$, the increase of risk of dysfunction of catheter and coagulation of the catheter in vitro. At present, in academic circle, it is generally believed that the condition is feasible and safe when post-filtration $\mathrm{iCa}^{2+}{ }^{2}$ is at $0.25 \mathrm{mmol} / \mathrm{L} \otimes 0.45 \mathrm{mmol} / \mathrm{L}$ and arterial $\mathrm{iCa}^{2+}$ is at $1.0 \mathrm{mmol} / \mathrm{L} \otimes 1.2 \mathrm{mmol} / \mathrm{L}^{[9-11]}$. We will compare the incidence of catheter dysfunction and hypocalcemia between the two locations in order to explore their clinical significance for anticoagulant safety.

In summary, in this randomized cross-over trial, we will hypothesized that compared with the SCV, when calcium was infused through the FV-VL, the arterial iCa ${ }^{2+}$ was lower. In addition, comparisons of the incidence of catheter dysfunction and hypocalcemia will help clarify which position is safer and more effective for calcium supplementation of calcium-free RCA-CVVH. 


\section{Declarations}

\section{Trial status}

Patient recruitment commenced in Dec. 2018 and is still ongoing. It is anticipated that recruitment will be completed in Oct. 2019.

\section{Ethics approval and consent to participate}

This study was approved by Medical Ethics Committee of the First Hospital of Lanzhou University (Number: LDYYLL2018-165) and registered at chictr.org.cn (ChiCTR1800020046). Written informed consent was signed by all participants.

\section{Consent for publication}

Not applicable.

\section{Availability of data and materials}

Not applicable.

\section{Competing interests}

The authors declare that there is no competing interests between the organization and the person in charge.

\section{Funding}

The authors declare that no funding has been secured for this study so far, including the design of the study or the collection, analysis, and interpretation of data or the writing of the manuscript.

\section{Authors' contributions}

JIANG conceived the study and design and drafted the protocol. ZHANG Zhigang conceived the study and design and commented on the protocol. JIN conceived the study and design and commented on the protocol. WANG commented on the protocol. WU commented on the protocol. ZHANG commented on the protocol. All authors have read and approved the manuscript, and ensure that this is the case.

\section{Acknowledgements}

The authors wish to acknowledge Pro. Jinhui Tian and Pro. Xiping Shen who provided advice based on their experience.

\section{Authors' information}

1, School of nursing, Lanzhou University, Lanzhou, 730000, P.R. China; 2, The First Hospital of Lanzhou University, Lanzhou, 730000, P.R. China 


\section{Abbreviations}

SCV: Subclavian vein; FV: Femoral vein; VL: The venous line of the hemofiltration catheter; VL-FL: The venous line of the hemofiltration catheter punctured in the femoral vein; RCA: Regional citrate anticoagulation; CRRT: Continuous renal replacement therapy; CVVH: Continuous venous-venous hemofiltration; $\mathrm{iCa}^{2+}$ : Calcium ion concentration; TMP: Transmembrane Pressure; PA: Access pressure; PV: Return pressure; $\mathrm{CVC}$ : Central venous catheter; $\mathrm{CaCl}_{2}$ : Calcium chloride; UFR: Ultrafiltration rate.

\section{References}

[1]Kidney Disease Improving Global Outcomes Kidney. KDIGO Clinical Practice Guideline for Acute Kidney Injury [J].Kidney Int,2012, 2(I):1-138.

[2]Liet J-M, Allain-Launay E, Gaillard-LeRoux B, et al. Regional citrate anticoagulation for pediatric CRRT using integrated citrate software and physiological sodium concentration solutions. Pediatric Nephrology.2014; 29(9):1625-1631.

[3]CAKARER S, EYUPOGLU E, GUNES C O, et al. Evaluation of the hemostatic effects of Ankaferd blood stopper during dental extractions in patients on antithrombotic therapy[J].Clin Appl Thromb Hemost,2013, 19 (1) : 96-99.

[4]IACOBAZZI V, INFANTINO V. Citrate-new functions for an old metabolite [J]. BiolChem, 2014,395(4):387399.

[5]Morabito S, Pistolesi V, Tritapepe L, et al. Regional citrate anticoagulation in cardiac surgery patients at high risk of bleeding: continuous veno-venous hemofiltration protocol with a low concentration citrate solution [J]. Critical Care, 2012, 16(3):R111.

[6] Yingfeng X, Suxia Z, Zhen guoYu, et al. Exploration of A Way to Replace Peripheral Blood Calcium Detection During RCA-HD [J]. Medical Innovation of China, 2016, 13(27):120-123

[7]Link A, Klingele M, Speer T, et al. Total-to-ionized calcium ratio predicts mortality in continuous renal replacement therapy with citrate anticoagulation in critically ill patients [J]. Crit Care, 2012, 16(3):1-11.

[8] Gubensek J, Orsag A, Ponikvar R, et , al. Calcium Mass balance during Citrate Hemodialysis: A Randomized Controlled Trial Comparing Normal and Low lonized Calcium Target Ranges[J]. PLOS ONE 11(12): e0168593.

[9] Tolwani A, Wille KM: Advances in continuous renal replacement therapy: Citrate anticoagulation update [J].Blood Purif, 2012, 34:88-93.

[10] Calatzis A, Toepfer M, Schramm W, Spannagl M, Schiffl H. Citrate anticoagulation for extracorporeal circuits: effects on whole blood coagulation activation and clot formation[J]. Nephron. 2001; 89: 233-6. 
[11] Yu Y, Peng S, Cen Z, et, al. Applying Regional Citrate Anticoagulation in Continuous Renal Replacement Therapy for Acute Kidney Injury Patients with Acute Liver Dysfunction: a Retrospective Observational Study[J]. Kidney Blood Press Res 2018,43: 1065-1074.

\section{Figures}

\begin{tabular}{|c|c|c|c|c|c|c|c|}
\hline & \multicolumn{7}{|c|}{ STYDY PERIOD } \\
\hline & \multirow{2}{*}{\begin{tabular}{|l|} 
Enrollment \\
Before CVVH \\
\end{tabular}} & \multirow{2}{*}{$\begin{array}{c}\text { Allocation } \\
2 \mathrm{H} \text { before } \\
\text { CWVH }\end{array}$} & \multicolumn{4}{|c|}{ Post-allocation } & \multirow{2}{*}{\begin{tabular}{|c|} 
Close-out \\
End of \\
data acquisition \\
\end{tabular}} \\
\hline TIMEPOINT & & & Before CWH & $\begin{array}{c}\text { CWVH in } \\
\text { stable period }\end{array}$ & Change pathways & $\begin{array}{c}\text { 1H after } \\
\text { Change pathways }\end{array}$ & \\
\hline \multicolumn{8}{|l|}{ ENROLLMENT } \\
\hline Eligibility screen & $x$ & & & & & & \\
\hline Informed consent & $x$ & & & & & & \\
\hline ALLOCATION & & $\mathrm{x}$ & & & & & \\
\hline \multicolumn{8}{|l|}{ INTERVENTION } \\
\hline$A: V L-S C V$ & & & & $x$ & $x$ & $x$ & \\
\hline B: SCV-VL & & & & $x$ & $x$ & $x$ & \\
\hline \multicolumn{8}{|l|}{ ASSESSMENT } \\
\hline Demographics & $\bar{x}$ & $\bar{x}$ & & & & & \\
\hline \multicolumn{8}{|l|}{ Related parameters of CVVH } \\
\hline B1ood flow & & & & $x$ & $\bar{x}$ & $x$ & \\
\hline Dosage of citrate & & & & $x$ & $x$ & $x$ & \\
\hline IMP & & & & $x$ & $\times$ & $x$ & \\
\hline PA & & & & $x$ & $x$ & $x$ & \\
\hline $\mathrm{PV}$ & & & & $x$ & $x$ & $x$ & \\
\hline \multicolumn{8}{|l|}{ UFR } \\
\hline \multicolumn{8}{|l|}{ B1ood gas analysis } \\
\hline $\mathrm{PH}$ & & & $x$ & $x$ & $\bar{x}$ & $x$ & \\
\hline iCa2+(Arterial Post-filtration) & & & $x$ & $x$ & $x$ & $x$ & \\
\hline $\mathrm{K}+$ & & & $x$ & $x$ & $x$ & $x$ & \\
\hline $\mathrm{tHbc}$ & & & $x$ & $x$ & $x$ & $x$ & \\
\hline Catheter dysfunction & & & & $x$ & & $x$ & $x$ \\
\hline Hypoca1 cemia & & & & $x$ & & $x$ & $x$ \\
\hline Reasons for discontinuation & & & & $x$ & & $x$ & $\times$ \\
\hline
\end{tabular}

\section{Figure 1}

Standard Protocol Items: Recommendations for Interventional Trials (SPIRIT) flow diagram: schedule of enrollment, interventions, and assessments. SCV: Subclavian vein; VL: The venous line of the hemofiltration catheter; $\mathrm{CVVH}$ : Continuous venous-venous hemofiltration; iCa2+: Calcium ion concentration; TMP: Transmembrane Pressure; PA: Access pressure; PV: Return pressure; UFR: Ultrafiltration rate; $\times$ :Perform the operation at this stage 


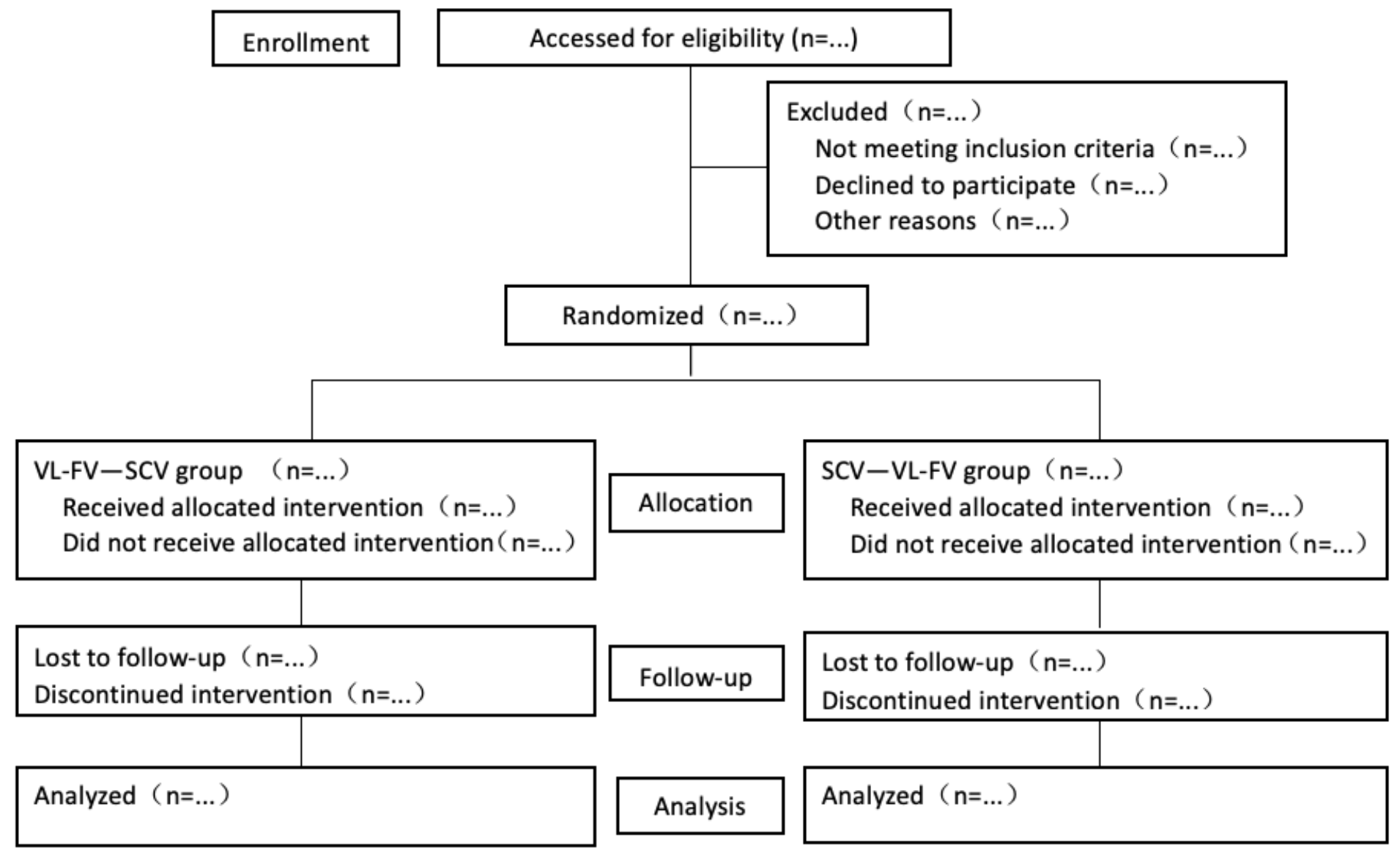

Figure 2

Consoladed standard of Reporting Trials (CONSORT) flow chart. SCV: Subclavian vein; VL-FL: The venous line of the hemofiltration catheter punctured in the femoral vein

\section{Supplementary Files}

This is a list of supplementary files associated with this preprint. Click to download.

- supplement1.pdf 